\title{
The danger signal plus DNA damage two- hit hypothesis for chronic inflammation in COPD
}

\author{
Kazutetsu Aoshiba', Takao Tsuji', Kazuhiro Yamaguchi², Masayuki Itoh¹ and \\ Hiroyuki Nakamura ${ }^{1}$
}

Affiliations: 'Dept of Respiratory Medicine, Tokyo Medical University Ibaraki Medical Center, Ibaraki, and ${ }^{2}$ Comprehensive and Internal Medicine, Tokyo Women's Medical University Medical Center East, Tokyo, Japan.

Correspondence: K. Aoshiba, Dept of Respiratory Medicine, Tokyo Medical University Ibaraki Medical Center, 3-20-1, Chuou, Ami, Inashiki, Ibaraki 300-0395, Japan. E-mail: kaoshibaQtokyo-med.ac.jp

ABSTRACT Inflammation in chronic obstructive pulmonary disease (COPD) is thought to originate from the activation of innate immunity by a danger signal (first hit), although this mechanism does not readily explain why the inflammation becomes chronic. Here, we propose a two-hit hypothesis explaining why inflammation becomes chronic in patients with COPD. A more severe degree of inflammation exists in the lungs of patients who develop COPD than in the lungs of healthy smokers, and the large amounts of reactive oxygen species and reactive nitrogen species released from inflammatory cells are likely to induce DNA double-strand breaks (second hit) in the airways and pulmonary alveolar cells, causing apoptosis and cell senescence. The DNA damage response and senescence-associated secretory phenotype (SASP) are also likely to be activated, resulting in the production of pro-inflammatory cytokines. These pro-inflammatory cytokines further stimulate inflammatory cell infiltration, intensifying cell senescence and SASP through a positive-feedback mechanism. This vicious cycle, characterised by mutually reinforcing inflammation and DNA damage, may cause the inflammation in COPD patients to become chronic. Our hypothesis helps explain why COPD tends to occur in the elderly, why the inflammation worsens progressively, why inflammation continues even after smoking cessation, and why COPD is associated with lung cancer.

@ERSpublications

A vicious cycle, involving mutually reinforcing inflammation and DNA damage, may cause chronic inflammation in COPD http://ow.ly/nYW4u

Received: July 042012 | Accepted after revision: Dec 122012 | First published online: Feb 082013

Support statement: This work was supported by a Grant-in-Aid for Scientific Research from the Ministry of Education, Science, and Culture, Japan, and by the Ministry of Health, Labour, and Welfare of Japan for the investigation of intractable diseases.

Conflict of interest: None declared.

Copyright @ERS 2013 


\section{Introduction}

Inflammation in chronic obstructive pulmonary disease (COPD) is currently understood as consisting of two stages: an innate immunity stage, in which the inflammation is activated by a danger signal (damageassociated molecular patterns (DAMPs)), and a subsequent acquired immunity stage $[1,2]$. The activation of the immune response and subsequent progression from innate immunity to acquired immunity is consistent with the natural disease course in smokers who develop the symptoms of COPD and in patients with mild disease who subsequently develop severe disease. However, this model does not readily explain why the inflammation in patients with COPD becomes chronic (unlike the situation in healthy smokers), why the inflammation in COPD patients progressively worsens, or why the inflammation persists in COPD patients even after they have stopped smoking.

Since pulmonary inflammation and lung ageing are closely associated, COPD is often referred to as a disease of accelerated or abnormal lung ageing, or a disease of lung inflammaging [3-6]. Lung ageing has been suggested to result from the time-dependent accumulation of damage to cells and macromolecules [3-6], whereas DNA damage is regarded as one type of damage that has been implicated in ageing [7]. In this article, we will review evidence supporting a concept in which DNA damage, such as DNA double-strand breaks, is involved in a mechanism through which the inflammation that occurs in COPD may subsequently become chronic. Based on this evidence, we propose a two-hit hypothesis in which inflammation initiated by a "danger signal" (first hit) and persistent inflammation arising from DNA damage (second hit) are involved in the development of COPD.

\section{Stepwise activation of the immune response in COPD}

Cosio et al. [1] suggested that the immune response is activated in a stepwise manner in the lungs of patients with COPD. During the activation of innate immunity in step 1, a danger signal, such as the highmobility group box 1 protein, ATP, or uric acid (also known collectively as DAMPs [8]), is released by cells that have been damaged by tobacco smoke (necrotic cells); this danger signal can activate a variety of receptors including Toll-like receptors, the receptor for advanced glycation end products, purinergic receptors and receptors within the inflammasome. The activation of such receptors in turn stimulates the production of interleukin (IL)- $1 \alpha$ and IL- $1 \beta$, thereby activating neutrophils and macrophages [2]. Step 2 represents a transitional phase from innate immunity to acquired immunity; during this step, CD $4^{+} \mathrm{T}$-cells and $\mathrm{CD}^{+}{ }^{+}$-cells proliferate and undergo differentiation and activation in response to dendritic cells presenting antigens released by necrotic cells and tissues. When immune tolerance during step 2 is insufficient, a transition to the activation of acquired immunity is thought to occur (step 3), resulting in severe inflammation and tissue damage in response to the activities of cytotoxic $\mathrm{CD}^{+} \mathrm{T}$-cells, T-helper (Th)1 CD4 ${ }^{+}$T-cells, Th17 CD4 ${ }^{+}$T-cells and B-cells. Cosio et al. [1] stated that in many smokers the process proceeds no further than step 1 , during which the activation of innate immunity occurs. In patients who develop COPD, however, the process proceeds from step 1 to step 2, resulting in an increase in disease severity and the subsequent activation of acquired immunity in step 3 [1].

This theory of the stepwise activation of the immune response is consistent with data obtained in smokers who develop COPD and suggests a progressive pathology in which patients who are mildly ill subsequently become severely ill. Nevertheless, the above theory does not address numerous questions. 1) Why is COPD more common in the elderly? 2) Why does the inflammation that occurs in COPD worsen progressively? 3) Why do steroids tend to be ineffective except in exacerbated cases? 4) Why does lung cancer tend to develop as a complication of COPD? 5) Why does the inflammation in COPD persist even after smoking cessation? For example, the theory of stepwise activation of the immune response suggests the involvement of an autoimmune response as the reason why inflammation persists after smoking cessation; unfortunately, the research performed to date has provided inconsistent results [1, 2, 9-12]. We believe an alternate mechanism that addresses the above-mentioned questions may depend on DNA damage, and not simply on the stepwise activation of the immune response. Specifically, cumulative DNA damage may explain why the inflammation in patients with COPD becomes chronic, and a considerable amount of accumulated data supports an association between chronic inflammation and DNA damage.

\section{Induction of DNA damage by chronic inflammation-induced reactive oxygen species and reactive nitrogen species}

Chronic inflammation is widely known to induce carcinogenesis by causing DNA damage [13]. For example, infection by Helicobacter pylori, hepatitis viruses and human papillomavirus is known to cause stomach cancer, liver cancer and cervical cancer, respectively [14]. Even in chronic inflammation cases without an infection-related cause, high rates of cancer have been reported (for example, colorectal cancer associated with ulcerative colitis and Crohn's disease, lung cancer and mesothelioma associated with inflammation caused by asbestos exposure, and lung cancer associated with COPD) [13, 15]. The 
mechanisms thought to underlie these relationships include the production of reactive oxygen species (ROS) and reactive nitrogen species (RNS), which can cause serious DNA damage including oxidation, nitration and double-strand breaks, by inflammatory cells [13, 16-19].

\section{Induction of chronic inflammation by DNA damage}

DNA damage is a cause of apoptosis and cell senescence, as well as a cause of carcinogenesis, and DNA damage has recently begun to attract attention as a cause of chronic inflammation [20-26]. For example, cells that have senesced as a result of DNA double-strand breaks produce many phlogogenic substances (e.g. IL-1 $\alpha$, IL-6, IL-8, granulocyte-macrophage colony-stimulating factor, growth-regulated oncogene $\alpha$, monocyte chemotactic protein (MCP)-2, MCP-3, and matrix metalloproteinases-1, -2, -3, -12, -13 and -14) [21-26]. The senescence-associated secretory phenotype (SASP) has been used to describe senescent cells that produce these phlogogenic substances [27]. Several molecular mediators are thought to contribute to this phenotype, including ATM, MDC1, NBS1, 53BP1, BRCA1 and Chk2; these mediators are activated as a result of DNA double-strand breaks and transmit the DNA damage response, which activates nuclear factor (NF)- $\mathrm{B}$ [23-26]. The inhibition of NF- $\kappa \mathrm{B}$ in progeroid mice has been shown to delay DNA damage-induced inflammation, cell senescence and age-related pathologies in multiple organs [28]. The pro-inflammatory cytokines produced in response to the activation of the DNA damage response have been shown not only to activate inflammatory cells, but also to intensify cell senescence and the SASP through the autocrine and paracrine actions of IL-1, IL-6 and IL-8 [23-26, 29]. Moreover, in vivo animal experiments have shown that colitis becomes chronic in $\mathrm{Atm}^{-/-}$mice, which have a reduced ability to repair DNA double-strand breaks compared with control $\mathrm{Atm}^{+/+}$mice [30]. Thus, recent studies support the concept that DNA damage induces chronic inflammation.

Summarising the above research findings, the following positive-feedback loop could be responsible for the chronic inflammation that develops in COPD: inflammatory cell activation $\rightarrow$ production of ROS/RNS $\rightarrow$ DNA double-strand breaks $\rightarrow$ activation of the DNA damage repsonse $\rightarrow$ induction of cell senescence $\rightarrow$ SASP $\rightarrow$ pro-inflammatory cytokine production $\rightarrow$ inflammatory cell activation $[23,24]$. Through the activation of this positive-feedback loop, a vicious cycle is formed in which chronic inflammation induces DNA damage; if not repaired, this DNA damage in turn intensifies the inflammation [23, 24]. In COPD, DNA damage may arise from the ROS or RNS released by inflammatory cells as well as the ROS present in tobacco smoke [31]. In fact, accumulating evidence suggests the occurrence of DNA damage in COPD [32-42].

DNA damage and SASP as a persistent source of inflammatory mediators in COPD Many investigators, including our own group, have reported the occurrence of DNA damage (including DNA oxidation and methylation, microsatellite DNA instability, loss of heterozygosity, and DNA doublestrand breaks) in the lung tissue of COPD patients [32-38]. DNA breaks have also been observed in peripheral blood lymphocytes $[40,43]$. The ROS present in tobacco smoke and the ROS or RNS produced by inflammatory cells are thought to be important causes of this type of DNA damage in COPD [42].

Fluorescent immunostaining using an anti- $\gamma \mathrm{H} 2 \mathrm{AX}$ antibody has made it possible to detect DNA doublestrand breaks with a high sensitivity [44]. Our recent studies using human lung tissue have revealed that: 1) ROS-mediated DNA double-strand breaks not observed in control smokers can be detected in the lung tissues of COPD patients, and 2) DNA double-strand breaks not only induce apoptosis and cell senescence, but also chronic inflammation via NF- $\mathrm{\kappa B}$-dependent pro-inflammatory cytokine production by senescent cells (SASP) [38, 43, 45]. Similarly, AMSELLEM et al. [46] showed that senescent pulmonary vascular endothelial cells produce phlogogenic substances, such as IL-6, IL-8, MCP-1 and soluble intercellular adhesion molecule-1, in COPD patients. Moreover, using animal experiments we showed that when DNA damage is specifically induced in mouse club cells (Clara cells), club cell senescence and chronic airway inflammation develop in association with the production of pro-inflammatory cytokines by the senescent club cells [47]. YAO et al. [48] also showed that activation of the anti-ageing sirtuin 1/FOXO3 pathway alleviated cell senescence, inflammation and emphysema in mice. Collectively, these results suggest the involvement of DNA damage, cell senescence and SASP in the pathogenesis of COPD.

\section{The mechanism by which inflammation becomes chronic in COPD: danger signal plus DNA damage, or the two-hit hypothesis}

Based on the data mentioned above, we have proposed a two-hit hypothesis explaining how inflammation becomes chronic in patients with COPD (fig. 1). The two-hit hypothesis consists of the initiation of inflammation by a danger signal and the perpetuation of inflammation as a result of DNA damage. As previously described by Cosio et al. [1], we considered the activation of innate immunity by a danger signal (first hit) to be the initial trigger leading to inflammation in smokers. Because of a genetic predisposition that facilitates the development of inflammation, more severe inflammatory cell infiltration occurs in the 
lungs of "susceptible" smokers who have a stronger predisposition for COPD than healthy smokers. Because of a less active antioxidant defence system in "susceptible" smokers, the large amounts of ROS or RNS released by the inflammatory cells and the ROS that is already present in tobacco smoke together induce double-strand breaks (second hit) in the DNA of the airway and alveolar cells. As a result, apoptosis and cell senescence are induced in the airways and alveolar cells, and the DNA damage response and SASP are activated, leading to the production of pro-inflammatory cytokines. These pro-inflammatory cytokines

a)

DNA damage (hit 2)

DDR-induced inflammation, apoptosis, senescence

Danger signal (hit 1)

DAMP-induced inflammation

Initiation of smoking

Progression to COPD

b)

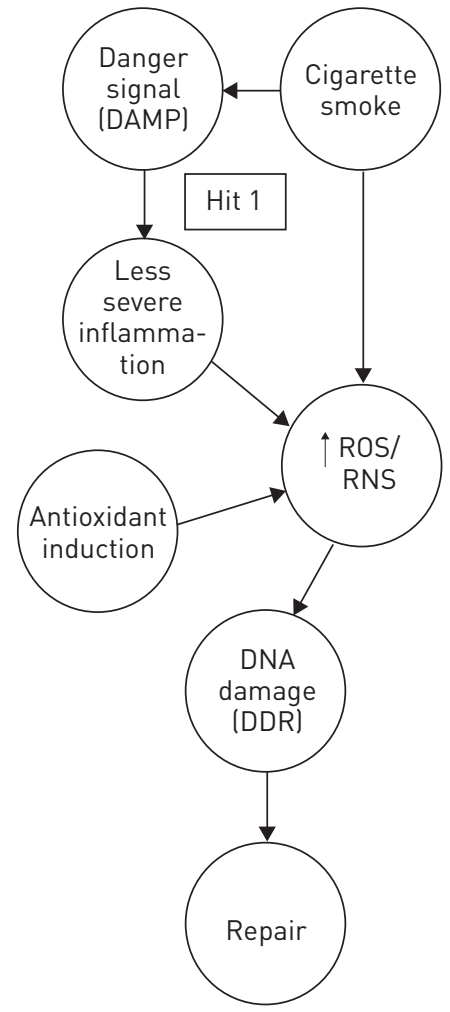

Susceptible smokers who are predisposed to COPD

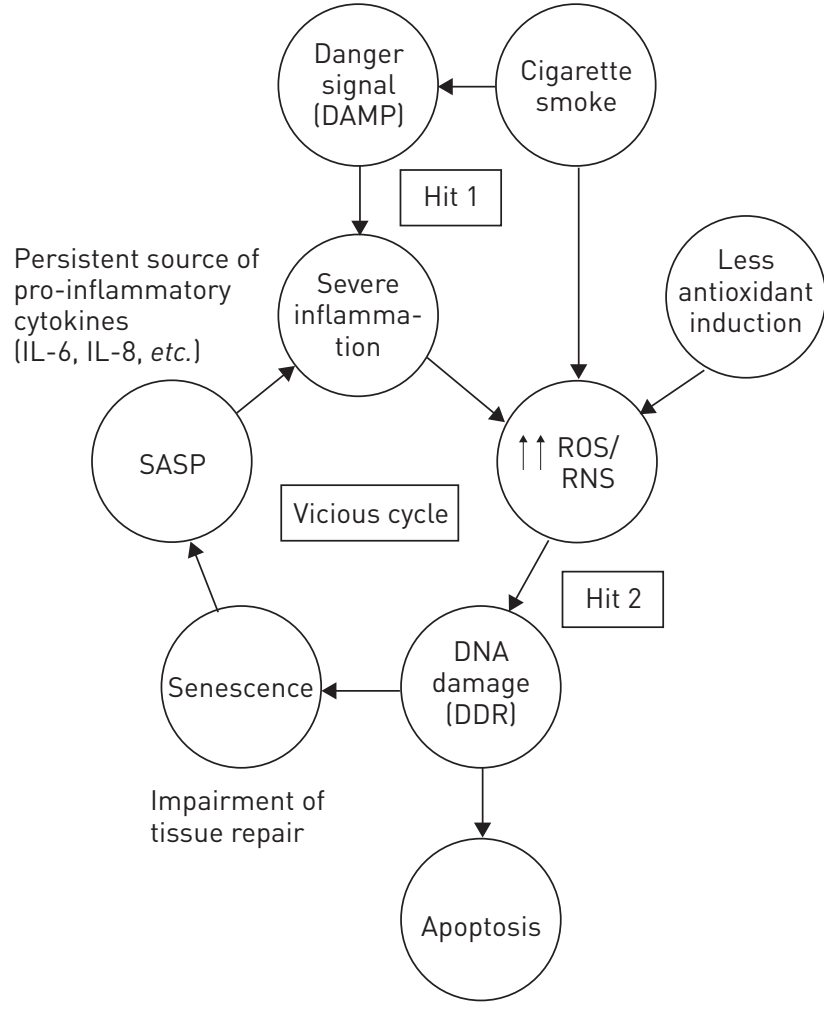

Loss of alveolar wall (emphysema)

FIGURE 1 Two-hit hypothesis, consisting of a danger signal plus DNA damage, explaining chronic inflammation in chronic obstructive pulmonary disease (COPD). a) In smokers, the danger signal (hit 1) is the trigger initiating inflammation in the lungs (the hypothesis of Cosio et al. [1]). According to the two-hit hypothesis, "susceptible" smokers who are predisposed to COPD develop DNA damage (hit 2), which induces apoptosis and senescence and activates the DNA damage response (DDR) and senescence-associated secretory phenotype (SASP), leading to the production of proinflammatory cytokines. b) Unlike healthy smokers, "susceptible" smokers develop DNA damage (e.g. double-strand breaks) in the airways and alveolar cells, presumably because of severer inflammatory cell infiltration and larger amounts of reactive oxygen species (ROS)/ reactive nitrogen species (RNS) production, a less efficient induction of antioxidants, and/or more inefficient DNA repair mechanisms. This, in turn, results in the activation of the DDR, apoptosis, senescence, and SASP and the production of pro-inflammatory cytokines. The pro-inflammatory cytokines then intensify inflammatory cell infiltration, forming a positive-feedback loop that further induces ROS/RNS production and the activation of the DDR, apoptosis, senescence, and SASP. In this manner, a vicious cycle is established between inflammation and DNA damage, and the inflammation becomes chronic. DAMP: damage-associated molecular pattern; IL: interleukin. 
further stimulate the infiltration of inflammatory cells, forming a positive-feedback loop in which cell senescence is intensified by autocrine and paracrine actions and promotes pro-inflammatory cytokine production via the SASP. The inflammation in COPD patients is thought to become chronic because of the vicious cycle between inflammation and DNA damage that occurs as a result of this positive feedback. In contrast to "susceptible" smokers, healthy smokers do not develop DNA damage [32-35, 38], presumably because of a lower severity of inflammatory cell infiltration resulting in the production of smaller amounts of ROS or RNS, more efficient adaptation by antioxidant induction, and/or more efficient DNA repair mechanisms. Based on this hypothesis, we propose that DNA damage in a smoker is the determining event in the progression to COPD. In other words, COPD develops when genotoxic stress (DNA damage) is added to the cellular stress (danger signal) of tobacco smoke.

TZORTZAKI and SIAFAKAS [42] hypothesised that dendritic cells recognise alveolar cells carrying gene mutations (somatic mutation) that have developed as a result of oxidative DNA damage as being "nonself", resulting in the persistent activation of the host immune system; however, with our hypothesis, we consider the activation of the DNA damage response and SASP via DNA double-strand breaks to be the mechanism responsible for chronic inflammation. However, these two hypotheses do not conflict with one another: they both involve the concept that DNA damage causes inflammation to become chronic in COPD.

Other theories explaining why the inflammation in COPD becomes chronic have also been proposed. For example, autoimmunity may account for the chronic inflammation in COPD via its ability to amplify a positive-feedback loop characterised by the mutual reinforcement of inflammation and autoreactive conditions [1, 49]. Furthermore, chronic infection may account not only for inflammation in COPD becoming chronic, but also for the higher prevalence of COPD in the elderly, who have a reduced immune surveillance and a reduced control of unwanted immune responses [50, 51].

The following research is needed to test the validity of the two-hit hypothesis explaining chronic inflammation in COPD. First, the actual occurrence of DNA damage during an early stage of COPD will need to be confirmed. DNA damage has already been reported to occur in the lung tissue of COPD patients, but many of the reported results were obtained in studies that compared control smokers with patients who had moderate-to-severe COPD [32-36, 38]. If the occurrence of DNA damage is the determining event that separates healthy smokers from COPD patients, DNA damage should have occurred even in patients with mild COPD and should progress as the severity of COPD increases. Secondly, animal experiments to confirm that COPD-like lesions develop as a result of DNA damage should be performed. Although we have previously reported that chronic airway inflammation develops as a result of DNA damage in mouse club cells [47], whether DNA damage is the cause of pulmonary emphysema or airway remodelling should be examined using a long-term animal model. Possible animal models for such investigations could include models created by long-term smoke exposure, or using animals with a reduced DNA repair capacity, such as $\mathrm{Atm}^{-/-}$mice. Thirdly, whether the occurrence of DNA damage in COPD patients is simply due to an increase in DNA damage, a decreased ability to repair such damage or a combination of these two mechanisms must be investigated. Previous studies have reported that ROS inhibit DNA mismatch repair enzyme activity and that the expression of Ku86, a protein which is required for the non-homologous endjoining repair of DNA double-strand breaks, is decreased in COPD patients, suggesting a decreased ability to repair DNA damage in COPD $[52,53]$. Fourthly, whether other genetic changes besides DNA doublestrand breaks, such as histone deacetylation, microsatellite DNA instability, DNA methylation, telomere shortening and microRNA alterations, are involved in the vicious cycle between inflammation and DNA damage should be examined [6].

If the development of DNA damage is the determining event in whether a smoker develops COPD, as proposed in this hypothesis, the presence of DNA double-strand breaks might serve as a biomarker for the development of COPD. For example, the detection of DNA double-strand breaks using the anti- $\gamma \mathrm{H} 2 \mathrm{AX}$ antibody in epithelial cells obtained in sputum or bronchoalveolar lavage fluid samples, or in peripheral blood lymphocytes from smokers might be useful as an early detection method for COPD. In addition, chemopreventive agents that protect against DNA damage (such as chemicals, vitamins and other dietary substances) might be useful not only for the prevention of carcinogenesis, but also for the prevention of COPD.

\section{Questions regarding the two-hit model and the pathogenesis of COPD}

Based on the assumption of the two-hit hypothesis, questions regarding the pathogenesis of COPD can be answered as follows. 1) Why is COPD more common in the elderly? According to our hypothesis, COPD might be more common in the elderly because a long time is required for DNA damage to accumulate. This concept is shared by the mutation accumulation theory, which states: DNA damage that has gradually accumulated exerts its harmful effects in old age [54]. 2) Why does inflammation in COPD worsen progressively? Inflammation in COPD might worsen progressively because of the vicious cycle between 
inflammation and DNA damage. 3) Why do steroids tend to be ineffective except in exacerbated cases? The fact that steroids have no effect on DNA damage and a limited effect on SASP [55] might explain, at least in part, why steroids tend to be ineffective in most patients with COPD. 4) Why does lung cancer tend to develop as a complication of COPD? The tendency for lung cancer to develop as a complication of COPD might be explained by the fact that DNA damage and oxidative stress are involved in the pathogenesis of both COPD and lung cancer. 5) Why does the inflammation in COPD persist even after smoking cessation? Inflammation in COPD might persist even after smoking cessation because DNA damage caused by smoking persists for a long time even after smoking cessation [56, 57].

\section{Conclusion}

We have proposed a two-hit hypothesis suggesting that inflammation in COPD becomes chronic as a result of the initiation of inflammation by a danger signal (first hit) and the perpetuation of inflammation by DNA damage (second hit). This hypothesis can explain why COPD is more common in the elderly (accumulation of DNA damage). It can also explain why inflammation in COPD worsens progressively (vicious cycle between inflammation and DNA damage), why inflammation in COPD continues even after smoking cessation (persistence of DNA damage), and why lung cancer can occur as a complication of COPD (DNA damage and oxidative stress contribute to both COPD and lung cancer).

\section{Acknowledgements}

The authors acknowledge S.I. Rennard (Pulmonary, Critical Care, Sleep and Allergy Section, University of Nebraska Medical Center, Omaha, NE, USA) for his critical review of the manuscript.

\section{References}

1 Cosio MG, Saetta M, Agusti A. Immunologic aspects of chronic obstructive pulmonary disease. N Engl J Med 2009; 360: 2445-2454.

2 Brusselle GG, Joos GF, Bracke KR. New insights into the immunology of chronic obstructive disease. Lancet 2011; 378: 1015-1026.

Ito K, Barnes PJ. COPD as a disease of accelerated lung aging. Chest 2009; 135: 173-180.

Faner R, Rojas M, MacNee W, et al. Abnormal lung aging in chronic obstructive pulmonary disease and idiopathic pulmonary fibrosis. Am J Respir Crit Care Med 2012; 186: 306-313.

Tuder RM, Petrache I. Pathogenesis of chronic obstructive pulmonary disease. J Clin Invest 2012; 122: 2749-2755.

Yao H, Rahman I. Role of histone deacetylase 2 in epigenetics and cellular senescence: implications in lung inflammaging and COPD. Am J Physiol Lung Cell Mol Physiol 2012; 303: L557-L566.

7 Hasty P, Campisi J, Hoeijmakers J, et al. Aging and genome maintenance: lessons from the mouse? Science 2003; 299: 1355-1359.

Matzinger P. The danger model: a renewed sense of self. Science 2002; 296: 301-305.

Lee SH, Goswami S, Grudo A, et al. Antielastin autoimmunity in tobacco smoking-induced emphysema. Nat Med 2007; 13: 567-569.

10 Cottin V, Fabien N, Khouatra C, et al. Anti-elastin autoantibodies are not present in combined pulmonary fibrosis and emphysema. Eur Respir J 2009; 33: 219-221.

11 Feghali-Bostwick CA, Gadgil AS, Otterbein LE, et al. Autoantibodies in patients with chronic obstructive pulmonary disease. Am J Respir Crit Care Med 2008; 177: 156-163.

12 Núñez B, Sauleda J, Antó JM, et al. Anti-tissue antibodies are related to lung function in chronic obstructive pulmonary disease. Am J Respir Crit Care Med 2011; 183: 1025-1031.

Cousssens LM, Werb Z. Inflammation and cancer. Nature 2002; 420: 860-867.

14 Internal Agency for Research on Cancer. Chronic infections. In: Stewart BW, Kleihues P, eds. World Cancer Report 2003. Lyon, IARC Press, 2003; pp. 56-61.

15 Yao H, Rahman I. Current concepts on the role of inflammation in COPD and lung cancer. Curr Opin Pharmacol 2009; 9: 375-383.

16 Marnett LJ. Oxyradicals and DNA damage. Carcinogenesis 2000; 21: 361-370.

17 Lonkar P, Dedon PC. Reactive species and DNA damage in chronic inflammation: reconciling chemical mechanisms and biological fates. Int J Cancer 2011; 128: 1999-2009.

18 Hiraku Y, Kawanishi S, Ichinose T, et al. The role of iNOS-induced DNA damage in infection- and asbestosinduced carcinogenesis. Ann NY Acad Sci 2010; 1203: 15-22.

19 Murata $\mathrm{M}$, Thanan $\mathrm{R}$, Ma N, et al. Role of nitrative and oxidative DNA damage in inflammation-induced carcinogenesis. J Biomed Biotechnol 2012; 2012: 623019.

20 Sancar A, Lindsey-Boltz LA, Unsal-Kaçmaz K, et al. Molecular mechanisms of mammalian DNA repair and the DNA damage check points. Annu Rev Biochem 2004; 73: 39-85.

21 Acosta JC, O'Loghlen A, Banito A, et al. Chemokine signaling via the CXCR2 receptor reinforces senescence. Cell 2008; 133: 1006-1018.

22 Kuilman T, Michaloglou C, Vredeveld LC, et al. Oncogene-induced senescence relayed by an interleukin-dependent inflammatory network. Cell 2008; 133: 1019-1031.

23 Kuilman T, Michaloglou C, Mooi WJ, et al. The essence of senescence. Genes Dev 2010; 24: 2463-2479.

24 Freund A, Orjalo AV, Desprez PY, et al. Inflammatory networks during cellular senescence: causes and consequences. Trends Mol Med 2010; 16: 238-246.

25 Rodier F, Coppé JP, Patil CK, et al. Persistent DNA damage signalling triggers senescence-associated inflammatory cytokine secretion. Nat Cell Biol 2009; 11: 973-979.

26 Rodier F, Campisi J. Four faces of cellular senescence. J Cell Biol 2011; 192: 547-556. 

functions of oncogenic RAS and the p53 tumor suppressor. PLoS Biol 2008; 6: 2853-2868.

28 Tilstra JS, Robinson AR, Wang J, et al. NF- $\kappa \mathrm{B}$ inhibition delays DNA damage-induced senescence and aging in mice. J Clin Invest 2012; 122: 2601-2612.

29 Orajo AV, Bhaumik D, Gengler BK, et al. Cell-surface-bound IL-1 $\alpha$ is an upstream regulator of the senescenceassociated IL-6/IL-8 cytokine network. Proc Natl Acad Sci USA 2009; 106: 17031-17036.

30 Westbrook AM, Schiestl RH. Atm-deficient mice exhibit increased sensitivity to dextran sulfate sodium-induced colitis characterized by elevated DNA damage and persistent immune activation. Cancer Res 2010; 70: 1875-1884. Toyooka T, Ibuki Y. Cigarette sidestream smoke induces phosphorylated histone H2AX. Mutat Res 2009; 676: 34-40. Siafakas NM, Tzortzaki EG, Sourvinos G, et al. Microsatellite DNA stability in COPD. Chest 1999; $116: 47-51$.

Zervou MI, Tzortzaki EG, Makris D, et al. Differences in microsatellite DNA level between asthma and chronic obstructive pulmonary disease. Eur Respir J 2006; 28: 472-478.

34 Samara KD, Tzortzaki EG, Neofytou E, et al. Somatic DNA alterations in lung epithelial barrier cells in COPD patients. Pulm Pharmacol Ther 2010; 23: 208-214.

35 Deslee G, Woods JC, Moore C, et al. Oxidative damage to nucleic acids in severe emphysema. Chest 2009; 135: 965-974.

36 Pastukh VM, Zhang L, Ruchko MV, et al. Oxidative DNA damage in lung tissue from patients with COPD is clustered in functionally significant sequences. Int J Chron Obstruct Pulmon Dis 2009; 6: 209-217.

37 Qiu W, Baccarelli A, Carey VJ, et al. Variable DNA methylation is associated with chronic obstructive pulmonary disease and lung function. Am J Respir Crit Care Med 2012; 185: 373-381.

38 Aoshiba K, Zhou F, Tsuji T, et al. DNA damage as a molecular link in the pathogenesis of COPD in smokers. Eur Respir J 2012; 39: 1368-1376.

39 Shi Y, Cao J, Gao J, et al. Retinoic acid-related orphan receptor- $\alpha$ is induced in the setting of DNA damage and promotes pulmonary emphysema. Am J Respir Crit Care Med 2012; 186: 412-419.

40 Ceylan E, Kocyigit A, Gencer M, et al. Increased DNA damage in patients with chronic obstructive pulmonary disease who had once smoked or been exposed to biomass. Respir Med 2006; 100: 1270-1276.

41 Maluf SW, Mergener M, Dalcanale L, et al. DNA damage in peripheral blood of patients with COPD. Mut Res 2007; 626: $180-184$.

42 Tzortzaki EG, Siafakas NM. A hypothesis for the initiation of COPD. Eur Respir J 2009; 34: $310-315$.

43 Tsuji T, Aoshiba K, Nagai A. Alveolar cell senescence exacerbates pulmonary inflammation in patients with chronic obstructive pulmonary disease. Respiration 2010; 80: 59-70.

44 Mah LJ, El-Osta A, Karagiannis TC. $\gamma \mathrm{H} 2 \mathrm{AX}$ as a molecular marker of aging and disease. Epigenetics 2010; 5: 129-136.

45 Aoshiba K, Nagai A. Senescence hypothesis for the pathogenetic mechanism of chronic obstructive pulmonary disease. Proc Am Thorac Soc 2009; 6: 596-601.

46 Amsellem V, Gary-Bobo G, Marcos E, et al. Telomere dysfunction causes sustained inflammation in chronic obstructive pulmonary disease. Am J Respir Crit Care Med 2011; 184: 1358-1366.

47 Zhou F, Onizawa S, Nagai A, et al. Epithelial cell senescence impairs repair process and exacerbates inflammation after airway injury. Respir Res 2011; 12: 78 .

48 Yao H, Chung S, Hwang JW, et al. SIRT1 protects against emphysema via FOXO3-mediated reduction of premature senescence in mice. J Clin Invest 2012; 122: 2032-2045.

49 Bolland S, Garcia-Sastre A. Vicious circle: systemic autoreactivity in Ro52/TRIM21-deficient mice. J Exp Med 2009; 206: 1647-1651.

50 Sethi S. Bacterial infection and the pathogenesis of COPD. Chest 2000; 117: 286S-291S.

51 Lee N, Shin MS, Kang I. T-cell biology in aging, with a focus on lung disease. J Gerontol A Biol Sci Med Sci 2012; 67: 254-263.

52 Chang CL, Marra G, Chauhan DP, et al. Oxidative stress inactivates the human DNA mismatch repair system. Am J Physiol Cell Physiol 2002; 283: C148-C154.

53 Caramori G, Adcock IM, Casolari P, et al. Unbalanced oxidant-induced DNA damage and repair in COPD: a link towards lung cancer. Thorax 2011; 66: 521-527.

54 Ljubuncic P, Reznick AZ. The evolutionary theories of aging revisited - a mini-review. Gerontology 2009; 55: 205-216.

55 Laberge RM, Zhou L, Sarantos MR, et al. Glucocorticoids suppress selected components of the senescenceassociated secretory phenotype. Aging Cell 2012; 11: 569-578.

56 Wistuba II, Lam S, Behrens C, et al. Molecular damage in the bronchial epithelium of current and former smokers. J Natl Cancer Inst 1997; 89: 1366-1373.

57 Mao L, Lee JS, Kurie JM, et al. Clonal genetic alterations in the lungs of current and former smokers. J Natl Cancer Inst 1997; 89: 857-862. 